Documentation et bibliothèques

DOCUMENTATION BIBLIOTHEQUES

\title{
Publications officielles québécoises - Le journal des Débats (III)
}

\section{Gaston Bernier}

Volume 22, numéro 4, décembre 1976

URI : https://id.erudit.org/iderudit/1055306ar

DOI : https://doi.org/10.7202/1055306ar

Aller au sommaire du numéro

Éditeur(s)

Association pour l'avancement des sciences et des techniques de la documentation (ASTED)

ISSN

0315-2340 (imprimé)

2291-8949 (numérique)

Découvrir la revue

Citer cet article

Bernier, G. (1976). Publications officielles québécoises — Le journal des Débats (III). Documentation et bibliothèques, 22(4), 183-187.

https://doi.org/10.7202/1055306ar

\section{Résumé de l'article}

Le journal des Débats de l'Assemblée nationale du Québec intéresse plusieurs catégories de personnes : législateurs, fonctionnaires, journalistes, étudiants, hommes d'affaires, cadres syndicaux et citoyens. Pour le consulter avec profit, il faut bien connaître ses différents index.
Tous droits réservés (c) Association pour l'avancement des sciences et des techniques de la documentation (ASTED), 1976
Ce document est protégé par la loi sur le droit d'auteur. L'utilisation des services d'Érudit (y compris la reproduction) est assujettie à sa politique d'utilisation que vous pouvez consulter en ligne.

https://apropos.erudit.org/fr/usagers/politique-dutilisation/ 


\title{
Publications officielles québécoises - Le journal des Débats (III)
}

\author{
Gaston Bernier \\ Bibliothèque nationale \\ Abidjan \\ Côte d'Ivoire
}

Le journal des Débats de l'Assemblée nationale du Québec intéresse plusieurs catégories de personnes: législateurs, fonctionnaires, journalistes, étudiants, hommes d'affaires, cadres syndicaux et citoyens. Pour le consulter avec profit, il faut bien connaitre ses différents index.

The journal des Débats of the Quebec National Assembly is of interest to many groups: legislators, civil servants, journalists, students, business men, union leaders and citizens. In order to use it efficiently it is necessary to understand its different indexes.

El periódico del debate de la Asamblea nacional de Québec interesa a varias categorías de gente: legis/adores, funcionarios, periodistas, estudiantes, hombres de negocio, cuadros sindicales y citadinos. Por consultarlo con provecho, hay que conocer sus diferentes indices.

L'existence généralisée des comptes rendus des débats parlementaires dans les pays démocratiques constitue une preuve, sinon un indice, de leur utilité ?

De même, une déduction similaire peut découler des coûts inhérents à l'enregistrement des délibérations et à leur publication. Au Québec, le journal des Débats a coûté à l'État, en 1974/1975, la jolie somme de $\$ 1,540,000^{2}$. II ne serait pas normal qu'un tel investissement soit fait inutilement. De plus, les crédits accordés au Bureau du journal des Débats pour 1976/1977 sont de l'ordre de $\$ 2,180,000$ alors que, pour la même année, le budget de la bibliothèque de l'Assemblée nationale atteindra $\$ 1,209,000^{3}$. Et encore faut-il souligner que le tirage du journal des Débats n'atteint pas 4,000 exemplaires!

\footnotetext{
1. Voir à cet effet les inventaires énumérés précédemment dans Documentation et bibliothèques, vol. 20 , no 4 (décembre 1974), 173 et vol. 21 , no 4 (décembre 1975), 200.

2. Comptes publics de la province de Québec pour l'année financière terminée le 31 mars 1975..., Québec, Éditeur officiel, 1976, vol. 1, p. 140.
}

3. Crédits pour l'année financière se terminant le 31 mars $1977 \ldots$.. Québec, 1976, p. 6-4.
On veut bien croire que le journal des Débats soit utile. Encore faut-il préciser en quoi et à qui il peut servir.

\section{Utilité}

Les comptes rendus verbatim des assemblées législatives servent d'abord et avant tout aux législateurs. Le député a besoin du journal des Débats pour faire la critique d'un opposant, pour établir la concordance entre ce qu'il a voulu dire et ce qu'il a dit et même pour mousser sa publicité chez ses commettants.

Le rôle du compte rendu quant à ces trois points est si vrai que le député reçoit automatiquement dix copies du journal des Débats. Ordinairement, il en garde un exemplaire et demande d'expédier les neuf autres à des personnes ou à des organismes dont il fournit la liste 4.

Les comptes rendus verbatim des assem-

4. Les bibliothécaires ne devraient pas hésiter à communiquer avec le député de leur circonscription et à lui demander de faire expédier à l'institution le journal des Débats si une telle publication répond aux besoins locaux. 
blées législatives constituent une source importante de la jurisprudence parlementaire. L'article 3 du Règlement de l'Assemblée nationale stipule que «la procédure... est réglée:...5e) par les précédents établis par suite de l'interprétation des lois et du règlement».

Ainsi donc si les "Hansard" sont essentiels au travail des parlementaires, on devra les trouver de toute nécessité dans les bibliothèques législatives. Et, effectivement, ces dernières en possèdent d'imposantes collections.

Le compte rendu est un instrument essentiel du haut fonctionnarisme et, en particulier, des fonctionnaires chargés de la rédaction des lois et des règlements. J. Peter Swann, dans un rapport signalé précédemment ${ }^{5}$, a mis en lumière cette fonction. Les spécialistes chargés de rédiger les lois et les règlements veulent connaître la situation qui amène le législateur à voter une nouvelle loi et le gouvernement à adopter de nouveaux règlements. A cet effet, une collection du journal des Débats est essentielle.

Au Québec, une étude sommaire de la distribution des Débats confirme le rôle de cette publication. L'éditeur des Débats distribuait 3,582 copies en 1972 . De ce nombre, 967 exemplaires étaient destinés aux députés (et aux personnes dont ils avaient donné les adresses) et 718 allaient à des fonctionnaires ${ }^{6}$.

La présence de collections de comptes rendus parlementaires dans les bibliothèques gouvernementales est, à ce titre, tout à fait justifiée.

Un troisième groupe de personnes s'intéresse grandement à l'activité des assemblées législatives et, logiquement, aux comptes rendus de ces mêmes assemblées: ce sont les journalistes des média électroniques, de la presse écrite quotidienne et hebdomadaire...

Ce groupe cependant s'appuie beaucoup moins sur la collection de telle ou telle institution que sur la copie préliminaire distribuée moins d'une heure après le prononcé d'un discours. L'édition quotidienne publiée quarante-huit heures au moins après chaque séance, même si elle est distribuée à la presse, n'a pas la même utilité que l'édition préliminaire tirée à une centaine d'exemplaires. A Québec, près de la moitié des copies de cette

5. J. Peter Swann, A Report Relating to the Publication of the Proceedings of the Legislative Assembly of Alberta, s.I., 1972 , p. 38.

6. Débats de l'Assemblée nationale, vol. 12, no 43 (8 juin 1972), p. 1352, col. 1. édition est distribuée à la Tribune de la presse.

Les comptes rendus des Débats parlementaires constituent un champ d'exploration exceptionnel pour les étudiants de niveau collégial et universitaire. Les initiateurs des reconstitutions des débats du $19 \mathrm{e}$ siècle ${ }^{7}$, historiens pour la plupart, ont bien ressenti la lacune existante et ils ont parfois expliqué en long et en large les raisons qui les avaient amenés à établir rétrospectivement ces comptes rendus ${ }^{8}$. Pour résumer, on peut dire que la présence des "Hansard" abrège de beaucoup les efforts de ceux qui voudraient établir la biographie d'un homme politique: le "Hansard" en regroupera tous les discours dans une même collection et on pourra les retracer grâce aux index. Dans un autre domaine, celui de l'étude des lois, l'absence de cette même publication complique les recherches: d'abord directement, parce que le document de base n'existe pas; de plus indirectement parce que la littérature secondaire, elle-même conditionnée par la documentation de base, sera relativement rare. Les étudiants en sciences politiques, quant à eux, pourront réaliser une analyse du contenu des Débats parlementaires pour éclairer les positions politiques des partis et déterminer leur évolution?

Les Débats peuvent donc servir à un grand nombre d'étudiants dans plusieurs disciplines. Même des linguistes ont déjà scruté la publication afin d'y étudier la langue parlée de nos représentants. Malheureusement, l'édition quotidienne est déjà trop améliorée!10

L'importance des "Hansard" comme champ de recherche et d'exploration justifie leur présence dans les bibliothèques des collèges et des universités. Ils constituent des documents de première main, des sources primaires. Les efforts déployés pour acquérir une collection exhaustive et ordonnée d'une telle publication constituent un excellent investissement.

L'activité des parlements affecte l'ensemble de la population: sociétés commerciales

7. Voir Documentation et bibliothèques, vol. 21 , no 4 (décembre 1975), 200.

8. Elisabeth Nish, "Canadian Hansard 1841...", Debates of the Legislative Assembly of United Canada, Montréal, Presses de l'École des hautes études commerciales, 1970- , vol. 1, p. XI-XIII.

9. Maurice Duverger, Méthodes des sciences sociales, 3 e éd., Paris, P.U.F., 1964, p. 98

10. Les chercheurs pourraient cependant se servir des bandes magnétiques conservées à l'Assemblée nationale. 
et industrielles, associations professionnelles, syndicats et, finalement, tout citoyen. L'homme d'affaires désire connaître rapidement les décisions gouvernementales qui pourraient avoir une influence sur son entreprise. Les cadres syndicaux également. À la limite, les discussions des assemblées législatives touchent tous les citoyens sans exception.

Or, présentement, les journaux reproduisent de moins en moins d'extraits de discours ou de discussions des législateurs. On semble préférer le style du reportage libre. De plus, il arrive souvent que les journalistes s'attardent à un événement intéressant et négligent l'ensemble des travaux. La même remarque vaut pour la presse électronique. Cette évolution, effet ou cause de la publication des Débats parlementaires, en rend l'existence essentielle.

Malgré tout l'intérêt que l'on devrait accorder au journal des Débats, il reste que le nombre d'abonnés "payants" reste fort restreint; il semble même décroître: il était de 1,337 en $1971^{11}$, a à peine augmenté en $1972^{12}$ et, présentement, s'élève à seulement 1,045 , selon le service de distribution du journal. À la décharge des citoyens, il faut reconnaître qu'il n'est pas facile de lire quotidiennement ce compte rendu souvent volumineux, ou même d'en assumer la conservation. Le réseau des bibliothèques publiques devrait au moins remplir ce dernier rôle. ${ }^{13}$

\section{Utilisation}

II ne suffit pas seulement d'être convaincu de l'utilité des Débats parlementaires; encore faut-il savoir s'en servir efficacement. Pour y parvenir, le chercheur devra se familiariser avec les index, mais il devra aussi garder à l'esprit certaines notions et données.

Le journal des Débats comporte deux paginations. La première est celle du compte rendu des délibérations de l'Assemblée nationale. La seconde, précédée d'une lettre, s'applique aux débats ayant lieu en commissions parlementaires. Depuis 1971, la partie consacrée aux commissions est plus volumineuse

11. Débats de l'Assemblée nationale, vol. 11, no 42 (20 mai 1971), p. 1692 , col. 2

12. Ibid., vol. 12, no 43 (8 juin 1972), p. 1352, col. 7.

13. L'abonnement au journal des Débats est de $\$ 8.00$ par année. S'adresser au Service des documents parlementaires, Assemblée nationale, Québec. Les années 1966 à aujourd'hui sont encore disponibles. que celle consacrée à l'Assemblée nationale proprement dite.

Le chercheur devra toujours tenir compte du temps nécessaire à la transcription des délibérations, à la correction, à la publication et à la distribution du journal lui-même. Normalement, l'édition quotidienne du journal des Débats paraît le surlendemain de chaque séance et elle est aussitôt distribuée. Celle des délibérations des commissions de l'Assemblée nationale, quant à elle, paraît environ dix jours après la séance. II reste que ces délais peuvent être beaucoup plus longs pour le lecteur de Montréal ou de Chicoutimi que pour celui de l'Assemblée nationale. Des députés ont déjà souligné cette lacune qui, parfois, les affecte $^{14}$ et qui agace à l'occasion leurs électeurs ${ }^{15}$.

Enfin, le chercheur devra disposer d'une collection en ordre. Comme le Bureau du journal des Débats ne distribue pas l'édition reliée à l'extérieur de l'Assemblée nationale, il appartient à chacun, dans sa bibliothèque, de mettre en ordre et de faire relier sa collection et de se procurer les index essentiels ${ }^{16}$.

\section{Les index}

Les éditeurs du journal des Débats ont publié jusqu'à maintenant trois types d'index: les index annuels, un index cumulatif et des index thématiques. A l'Assemblée nationale, on dresse un index courant qui constitue la clé des délibérations de la session en cours.

a) Les index annuels. Le service de l'index publie après chaque session une table générale des débats ayant eu lieu au cours de la session. Ordinairement, le volume paraît deux ou trois mois environ après la prorogation de l'Assemblée nationale.

Jusqu'à maintenant, 15 index sessionnels ont été publiés ${ }^{17}$. Ils varient en importance selon la longueur de la session. L'un, celui de la 5 e session de la 27 e législature (1965)

14. Clément Vincent, in Débats de l'Assemblée nationale, vol. 12 , no 43 (8 juin 1972), p. 1351, col. 1.

15. Antoine Drolet, in Débats..., col. 12, no 40 (2 juin 1972), p. 1293, col. 1

16. Ceux-ci ne sont pas distribués automàtiquement aux abonnés du journal des Débats. Ils sont vendus séparément. On peut se les procurer en s'adressant au Service des documents parlementaires, Assemblée nationale du Québec, Québec. Prix: $\$ 2.00$ chacun.

17. 1964,1965 (2), 1966, 1966/67, 1968, 1969, 1970 (2), 1971, 1972/73, 1973 (2), 1974, 1975. Ces volumes sont encore disponibles. 
ne compte que six pages et est inséré dans le compte rendu lui-même. Dans tous les autres cas, les index sont publiés en fascicules séparés et comptent ordinairement plus de cent pages, parfois plus de deux cents. On a indexé le compte rendu de la $2 e$ session de la 28e législature (1967) mais l'index n'en a pas été publié. Par contre, le service a publié, sous forme de document polycopié, une table générale des délibérations de la 1ère session de la 27e législature (1963).

Depuis 1964, on a constamment amélioré l'index sessionnel tout en respectant le schéma original. II comporte deux parties: un index des noms des participants et un index des sujets débattus.

Dans l'ensemble la première partie est relativement plus volumineuse que la seconde. L'approche selon l'ordre alphabétique des députés est fort simple et permet d'échapper au problème des vedettes-matière. $P$ ar ailleurs, elle facilite la tâche des biographes et des personnes qui peuvent s'intéresser aux interventions de leur député.

Sous le nom d'un député, les auteurs procèdent selon l'ordre alphabétique des sujets sans tenir compte de la nature des interventions: question ou réponse, déclaration ministérielle, dépôt de document, etc. Cependant, un certain nombre d'abréviations permet d'en déterminer la nature.

La seconde partie de l'index sessionnel porte sur les sujets débattus à l'Assemblée nationale. Les descripteurs utilisés le sont avec une certaine régularité même si les auteurs de l'index se sentent relativement libres de leur fichier-autorité.

Quelques remarques s'imposent: 1) les activités d'un ministère sont indexées au nom du ministère; 2) les délibérations des commissions le sont sous la vedette "commissions parlementaires"; 3) l'étude des projets de loi fait l'objet d'une sous-section classée à "Projets de loi...»: on y retrouve aisément les grandes étapes des projets et les renvois au journal des Débats. A noter que les projets de loi publics sont énumérés par numéro d'ordre (et non par sujets) comme les projets de loi privés.

En plus de ces deux parties, l'index comprend certaines listes qui peuvent s'avérer utiles: membres du cabinet, adjoints parlementaires, haut personnel politique et administratif de l'Assemblée nationale, députés.
Depuis quelques années, on a même ajouté un calendrier sessionnel.

b) Le Service de l'index a également publié une table cumulative ${ }^{18}$ couvrant les quatre premières années du journal des Débats. Elle est encore disponible à l'heure présente chez l'éditeur. Malheureusement, on ne compte pas, pour le moment, publier un second volume du même type.

Cet index, même s'il peut être utile, n'est pas essentiel au même titre que les index annuels ou sessionnels.

La facture de ce volume est très particulière. Ce dernier est divisé en trois sections. On $y$ trouve d'abord un index alphabétique des principaux sujets abordés en Chambre (près de 400 entre 1964 et 1968). Dans un premier temps, les auteurs ont dressé une liste des sujets et, à la suite, la liste des mêmes descripteurs suivis de références et de certaines précisions. A noter que les références renvoient tantôt au journal des Débats, tantôt à l'index annuel. La seconde partie est constituée d'une liste des projets de loi publics déposés à l'Assemblée, énumérés par année et par numéro d'ordre. Les auteurs donnent le titre exact des projets et les renvois d'usage aux principales étapes de leur étude. Un index méthodique complète le volume. On y a classé sous cinq sections (politique, économique, sociale, religieuse et culturelle) les descripteurs utilisés dans la première partie de même que les projets de loi énumérés dans la seconde.

c) Le Bureau du journal des Débats publie enfin des tables thématiques et rétrospectives. Ces publications s'adressent évidemment à une clientèle restreinte. De fait, les deux premiers fascicules ${ }^{19}$ seront surtout utiles à des spécialistes.

Ces deux fascicules contiennent des références chronologiques allant de 1965 à 1973 dans le cas du Code des professions tandis que celles sur la réforme électorale couvrent la période qui va de 1961 à 1973. Évidemment, chaque événement est référé aux passages correspondants du journal des Débats. Ces

18. Débats de l'Assemblée nationale du Québec. Index cumulatif (1964 à 1968 incl.), Québec, 1969, 89 p.

19. Code des professions et lois connexes. Index 1965-73..., Québec, Assemblée nationale, Journal des Débats, Service de l'index, 1973, 17 p. (Index cumulatif; 1 ) et Réforme électorale, Québec, Assemblée nationale, Journal des Débats, Service de l'index, 1974, 52 p. (Index cumulatif; 2). Les deux fascicules sont épuisés. 
deux publications contiennent également la liste des projets de loi touchant les deux domaines et les références aux Débats. L'index des délibérations sur la réforme électorale est suivi de résumés analytiques des séances de la Commission de l'Assemblée nationale, d'une liste bibliographique, d'un bref index des sujets et d'un index des participants et experts. L'usager averti prendra rapidement conscience du fait que cette nouvelle série va au-delà de l'index traditionnel. Non seulement renvoie-t-on au journal des Débats, mais on fournit également de nombreux renseignements.

Le Service de l'index devrait publier le troisième cahier de la collection très bientôt. II portera sur les activités de la 27 e législature du Québec, celle qui fut élue à la fin de 1962 et qui fut dissoute en 1966 .

d) L'Index courant. Les index énumérés précédemment paraissent toujours avec un certain retard. Le journal des Débats ne publie pas, comme c'est le cas à Ottawa, un index cumulatif et périodique. L'usager ne possède donc pas d'index pour la session en cours; il lui faudra parfois feuilleter dix mille pages et près de 300 fascicules (session de 1975) sans autre instrument que la table des matières de chaque livraison.

Pour combler cette lacune, le Service de l'index permet la consultation du fichier établi quotidiennement au cours d'une session. Le chercheur intéressé peut entrer en communication avec le service de l'index par téléphone, par lettre ou encore en se rendant au Palais législatif ${ }^{20}$.

L'utilisation rationnelle du journal des Débats repose sur l'existence des index annuels et de l'index courant. Les index cumulatifs généraux ou spécialisés constituent également des instruments utiles aux chercheurs, sans répondre pour autant à des besoins essentiels.

L'enregistrement et la publication des Débats parlementaires a fait un long chemin depuis l'engagement de Thomas Gurney, en 1813, comme sténographe du Parlement de Grande-Bretagne ${ }^{21}$. Si, à l'origine, on enregistrait surtout les débats des Communes sié-

20. Par téléphone: (1-418)643-2771. Adresse: Service de l'index, Journal des Débats, Assemblée nationale, bureau 69-A, Québec.

21. A.R. Kennedy, "The Shorthand Writer to The Houses of Parliament", The Table, vol. 43 (1975), 58-60. geant à titre de tribunal (nous avons connu ici le même phénomène ${ }^{22}$ ) aujourd'hui on enregistre à peu près systématiquement ${ }^{23}$ les délibérations des assemblées parlementaires.

L'évolution continue. On remplace de plus en plus le sténographe par un magnétophone. $\mathrm{Ce}$ qui fait que les chercheurs ont un instrument supplémentaire à leur disposition quand la publication semble inadéquate. A l'Assemblée nationale, on conserve un enregistrement des délibérations. La publication elle-même des Débats parlementaires profite de la présence de l'ordinateur ${ }^{24}$. Un tel instrument permettrait aux éditeurs du journal des Débats d'insérer périodiquement dans la publication un tableau de l'état de la législation de même qu'une liste à jour des documents parlementaires rendus publics au cours de la session, comme on le fait en Alberta ${ }^{25}$.

La diffusion même des délibérations des assemblées parlementaires connaît une extension remarquable grâce à la radio et à la télévision. Les expériences se multiplient dans le monde ${ }^{26}$. Au Québec, le problème est posé et une sous-commission de la commission de l'Assemblée nationale doit se prononcer sur l'éventualité de la radiodiffusion et de la télédiffusion des débats. Même s'il y a peu de chances qu'on permette la transmission des délibérations de l'Assemblée nationale mais qu'on se satisfasse, pour le début, des séances des commissions parlementaires les plus importantes, déjà ces transmissions permettront une diffusion des travaux de nos parlementaires hors de portée du journal des Débats.

22. Par exemple, lors de la comparution du journaliste John H. Roberts in Journaux de l'Assemblée législative, vol. 57 (1922), p. 34

23. Au Québec, il semble que la seule exception soit celle des délibérations de la Commission des engagements financiers. Voir à ce sujet Débats de l'Assemblée nationale (commissions parlementaires), vol. 15, no 135 (17 juillet 1974), p. B-5691 B-5696.

24. L'expérience en la matière du Parlement du Canada est bien décrite dans Alexander Small, "The use of computers in bilingual publishing and retrieval of parliamentary publications". The Table, vol. 42 (1974), 66-72. On pourra lire également J. Peter Swann, "The computer and Hansard". The Parliamentarian, vol. 54, no. 4 (October 1973), 223-225.

25. Voir Alberta Hansard, vol. 2, no. 65 (18-19 October 1973).

26. Gaston Bernier, "Parlements, parlementaires et parlementarisme; bibliographie sélectiven, Bibliothèque de la Législature du Québec, Bulletin, vol. 5, no 1-2 (janvier-avril 1973), 70-71. 